\title{
Assessing usability of QIAreach QuantiFERON-TB platform in a high tuberculosis prevalence, low-resource setting
}
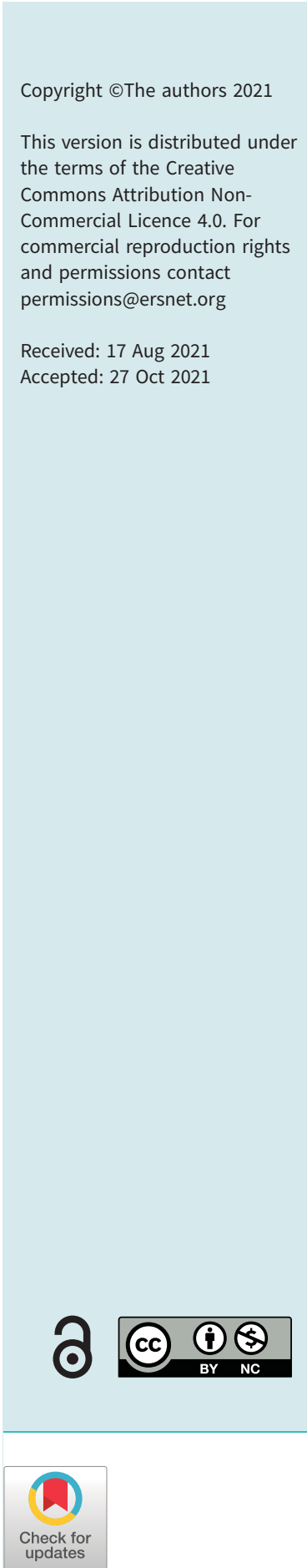

\section{To the Editor:}

The World Health Organization (WHO) estimates that 1.8 billion people, close to a quarter of the world's population are infected with Mycobacterium tuberculosis [1]. Despite substantial declines in tuberculosis (TB) incidence over the past decade, Zambia still has the seventh highest TB incidence in sub-Saharan Africa and remains one of the 30 WHO high TB-burden priority countries [2]. In 2019, there were 59000 new individuals with active TB disease in Zambia (incidence rate of 333 per 100000 per year), which resulted in 15400 TB-related deaths, of which $62 \%$ were among people living with HIV [2].

Management of TB infection represents a neglected component of national TB programmes [3]. A reliable diagnosis followed by successful TB preventive therapy is crucial to reach the End TB strategy goals and to eliminate TB $[4,5]$. Tuberculin skin test (TST) and interferon- $\gamma$ release assays (IGRAs) are the main diagnostic tools for TB infection diagnosis, however, both present strengths and limitations [6]. The TST requires two visits, is not easy to administer and is influenced by conditions, including bacille CalmetteGuérin vaccination, that can reduce the skin reactivity [7-9]. IGRAs were demonstrated to overcome the drawbacks of TST [10]. To date, two IGRA tests are recommended by the WHO: T-SPOT.TB (Oxford Immunotec, Abingdon, UK) and QuantiFERON-TB Gold Plus (QFT-Plus; QIAGEN, Hilden, Germany) [11].

The QFT-Plus is a new generation IGRA that uses innovative antigens that elicit both CD8 and CD4 T-cell responses, enabling a more comprehensive assessment of cell-mediated immune response to TB infection $[12,13]$. The need for established laboratory infrastructure and trained personnel represent one of the main barriers for the implementation of QFT-Plus in low-resource settings [14]. QIAGEN has recently developed a new lateral flow immunoassay for the diagnosis of TB infection, the QIAreach QuantiFERON-TB (QIAreach QFT) assay. The prototype QIAreach QFT is a semi-automated assay that uses the same TB2 tube as QFT-Plus to detect interferon- $\gamma$ in plasma released from both CD4 and CD8 T-cells. After incubation, without the need to perform enzyme-linked immunosorbent assay (ELISA), the sample is analysed on a portable platform, eHubs, performing up to eight tests in $20 \mathrm{~min}$, providing a final qualitative result (positive, negative, or error) $[15,16]$.

In this study we aimed to assess the usability of the prototype QIAreach QuantiFERON platform in a high TB prevalence, low-resource setting. We report the results of the prototype QIAreach QFT assay usability study conducted in 2019 across six laboratories in Zambia using whole blood collected from participants enrolled in the Tuberculosis Reduction through Expanded Antiretroviral Treatment and Screening (TREATS) infection cohort study.

The study was conducted across six laboratories situated in four provinces of Zambia. The six laboratories were the Zambart Central Laboratory located in Lusaka (Lusaka province) and five regional laboratories located in Livingstone (Southern province), Choma (Southern province), Kabwe (Central province), Ndola (Copperbelt province) and Kitwe (Copperbelt province). The selected laboratories were purposively and conveniently chosen due to their involvement with the TREATS infection cohort study in Zambia and are not representative of the total Zambian population. Usability of the prototype QIAreach QFT and training needs for the assay implementation were assessed across three domains: effectiveness, efficiency, and user satisfaction.

Shareable abstract (@ERSpublications)

QIAreach QuantiFERON-TB is a portable IGRA with the potential to improve accessibility of TB infection diagnosis in low-resource settings https://bit.ly/3nTzolf

Cite this article as: Kaaba C, Ruperez M, Kosloff B, et al. Assessing usability of QIAreach QuantiFERONTB platform in a high tuberculosis prevalence, low-resource setting. ERJ Open Res 2021; 7: 00511-2021 [DOI: 10.1183/23120541.00511-2021]. 
To achieve a valid preliminary data on usability, nine laboratory workers (two laboratory assistants, three laboratory scientists and four laboratory technicians) were purposively selected based on their job title and laboratory experience [17]. Operators were provided with the following: the QIAreach QFT eHub, a laptop computer with pre-installed QIAreach QFT software (optional; for connectivity with laboratory reporting systems only), QIAreach QFT test kits and diluent buffer, QIAreach QFT package insert, an adjustable standard laboratory pipette capable of dispensing $150 \mu \mathrm{l}$ and disposable pipette tips, and TB2 tube with harvested plasma. During the usability testing, operators were asked to read the instructions outlined in the package insert, and complete four tasks using both the eHubs and software. The operators did not receive any initial aid from the observer. If participants struggled with a task, the observer provided hints to help users through the process.

For each task, metrics on success (pass, fail, pass with hints), total task time, and ease of use rating (five-point Likert scale) were collected. Total task time is an indicator of user efficiency. Shorter task times on subsequent identical tasks are indicative of learnability. Common errors were collected after assay completion and categorised into those issues that prevent the user from completing the assay and those that increase the risk of human error during the task.

Written consent was obtained from eligible participants, who agreed to take part in the TREATS infection cohort at enrolment and there was no additional consent obtained for this study.

Ethics approval for the infection cohort was obtained from the Biomedical Research Ethics Committees of the University of Zambia, Lusaka, Zambia and the London School of Hygiene and Tropical Medicine, London, UK.

Overall task completion rate was $89-100 \%$. Table 1 provides a visual representation of the pass and fail rates of each task. One participant out of nine could not understand the software instructions in the QIAreach QFT package insert. However, the participant was able to complete the assay manually. Two participants had difficulty loading the sample on the QIAreach eStick. The average time from setup to results ranged from 22 to $40 \mathrm{~min}$. Users with experience performing the QFT-Plus assay completed the test faster than users without experience, 26 min versus $35 \mathrm{~min}$. Two participants were unable to adjust the pipette to the required volume of $150 \mu \mathrm{L}$. Two participants tried to mix the test sample up and down without the disposable tip on the pipette. One participant transferred the sample buffer twice and added insufficient plasma to the sample processing tube. Two participants added the test sample to the eStick sample port many times. One participant added wrong information in the software.

On average, participants had 7 years of laboratory experience. When asked about previous experience with IGRA technology, six operators reported experience using the QuantiFERON Gold-In-Tube/Plus.

The QIAreach QFT test provides a significant contribution to global TB infection diagnostics. The usability assessment of the prototype QIAreach QFT represents an important aspect that affects user acceptance and the introduction of a new test into the TB care cascade. In our study, we found that all participants obtained valid results and could use the platform without any problems or with little

\section{TABLE 1 Summary of task success rate, time from setup to results and user satisfaction by participant}

\begin{tabular}{|c|c|c|c|c|c|c|c|c|c|c|}
\hline & \multicolumn{9}{|c|}{ Participant } & \multirow[t]{2}{*}{ Effectiveness (i.e. pass rate) } \\
\hline & 1 & 2 & 3 & 4 & 5 & 6 & 7 & 8 & 9 & \\
\hline Task 1 & $\mathrm{P}$ & $\mathrm{P}$ & P & P & $\mathrm{P}$ & P & P & $\mathrm{P}$ & $P$ & 9/9 (100\%) \\
\hline Task 2 & $\mathrm{PH}$ & $\mathrm{PH}$ & $P$ & $P$ & $P$ & $\mathrm{P}$ & $\mathrm{P}$ & $\mathrm{P}$ & $P$ & $9 / 9(100 \%)$ \\
\hline Task 3 & $\mathrm{PH}$ & $\mathrm{PH}$ & $P$ & $P$ & $P$ & $\mathrm{P}$ & $\mathrm{P}$ & $\mathrm{P}$ & $P$ & $9 / 9(100 \%)$ \\
\hline Task 4 & $\mathrm{~F}$ & $\mathrm{PH}$ & $P$ & $P$ & $P$ & $P$ & $P$ & $P$ & $P$ & $8 / 9(89 \%)$ \\
\hline Efficiency ${ }^{\#}$, min & 40 & 36 & 30 & 30 & 26 & 23 & 29 & 26 & 22 & \\
\hline User satisfaction" & 2 & 4 & 4 & 4 & 5 & 5 & 4 & 5 & 4 & \\
\hline \multicolumn{11}{|c|}{$\begin{array}{l}\text { Task 1: setup (place blood tube on eHub, place estick in eHub, add processing tube on eHub); task 2: load } \\
\text { sample (add buffer to processing tube, remove plasma from blood tube, add plasma to processing tube, mix } \\
\text { plasma and buffer in processing tube); task 3: run test (add test sample to eStick port); task 4: process results } \\
\text { (enter and track data in software). P: pass; F: fail; PH: pass with hint; \#: mean time from setup to results; } \\
\text { ': Likert scale, 1=very unsatisfied, } 2=\text { =unsatisfied, } 3=\text { neutral, } 4=\text { =satisfied, } 5=\text { =very satisfied. }\end{array}$} \\
\hline
\end{tabular}


difficulties. Operators with prior experience with QFT-Plus completed the tasks faster than users without prior experience. Only one operator did not use the software due to his limited computer skills. The study also showed that seven operators out of nine correctly used a laboratory pipette without any difficulty. The key issue categorised as being at risk of human error when performing the assay was a lack of practice using the standard laboratory pipette. However, considering that the skills for using laboratory pipettes in low-resource settings are very limited, a disposable pipette and video training is recommended to reduce errors.

The primary driver of failed tasks was lack of expertise in using computers. High inconsistencies with the features of the device and those described in the instructions were found. Regardless of these challenges, an ease-of-use rating was assigned, with a majority of participants reporting ratings of 4 or 5 on a Likert scale for assay usability. When asked about satisfaction, the participants commented on the platform being user-friendly, the content being enough and the instructions being easy to understand and follow. The time taken for each user to perform the assay from the first to the last sample decreased as the user's experience and familiarity with the assay increased.

This study demonstrated that the prototype QIAreach QFT has a number of operational advantages compared with the more complex current IGRA assays. The prototype QIAreach QFT is an innovative digital lateral flow, battery operated system that replaces the laboratory-based ELISA workflow. The system also uses an optimised single tube workflow requiring $1 \mathrm{~mL}$ compared with the $4 \mathrm{~mL}$ needed for QFT-Plus and T-SPOT. The QIAreach QFT assay requires a time to results of 20 min compared with the $150 \mathrm{~min}$ needed for the ELISA-based detection assay. In addition, the QIAreach QFT allows eight samples to be run independently eliminating the need to batch samples and providing timely TB infection diagnosis.

The QIAreach QFT assay is suitable for implementation in remote areas, where limited infrastructure has hampered the accessibility of IGRA technologies to those in need. Further studies are needed to establish the assays performances as well as the feasibility of introducing this new assay at larger scale to improve TB control in regions with limited infrastructure.

\section{Conceptor Kaaba $\oplus^{1}$, Maria Ruperez ${ }^{2}$, Barry Kosloff ${ }^{1,2}$, Nduku Ndunda ${ }^{3}$, Kwame Shanaube ${ }^{1}$ and Helen Ayles ${ }^{1,2}$ on behalf of the TREATS Study Team}

${ }^{1}$ Zambart, Lusaka, Zambia. ${ }^{2}$ London School of Hygiene and Tropical Medicine, London, UK. ${ }^{3}$ Cepheid Middle East, a Danaher company FZ LLC, Dubai, United Arab Emirates.

Corresponding author: Conceptor Kaaba (conceptor@zambart.org.zm)

Acknowledgement: Authors would like to express their sincere appreciation and gratitude to all members of the TREATS infection cohort study team in Zambia, and to the study participants and their communities, and to the Zambart and non-Zambart laboratory staff, for their contributions to the research. The content herein is solely the responsibility of the authors.

Provenance: Submitted article, peer reviewed.

Author contributions: M. Ruperez, N. Ndunda and H. Ayles conceived and designed the study. C. Kaaba made substantial contributions to the acquisition of the data. C. Kaaba, B. Kosloff and N. Ndunda helped with analysis and interpretation of data. K. Shanaube and C. Kaaba took the lead in writing the paper with input from all authors. K. Shanaube, H. Ayles and C. Kaaba revised the paper critically for intellectual content. All authors were involved in the final approval of the version to be submitted.

Conflict of interest: Some of the authors (C. Kaaba, M. Ruperez, B. Kosloff, K. Shanaube and H. Ayles) declare receiving a grant from EDCTP for TREATS and one author (N. Ndunda) is a former employee of QIAGEN. QIAGEN (Hilden, Germany) provided the QIAreachTM QuantiFERON-TB test kits free of charge.

Support statement: QIAGEN (Hilden, Germany) provided the QIAreachTM QuantiFERON-TB test kits free of charge. QIAGEN had no role in study design, collection, analysis or interpretation of data. This project is part of the EDCTP2 programme supported by the European Union (grant number RIA2016S-1632-TREATS). Funding information for this article has been deposited with the Crossref Funder Registry. 
References

1 TB Alliance. TB is a Pandemic. www.tballiance.org/why-new-tb-drugs/global-pandemic

2 World Health Organization. Global Tuberculosis Report 2020. Geneva, World Health Organization, 2020. www. who.int/publications/i/item/9789240013131

3 Harries A, Kumar A, Satyanarayana S, et al. Treatment for latent tuberculosis infection in low- and middle-income countries: progress and challenges with implementation and scale-up. Expert Rev Respir Med 2020; 14: 195-208.

4 Chakaya JM, Harries AD, Marks GB. Ending tuberculosis by 2030 - pipe dream or reality? Int J Infect Dis 2020; 92: S51-S54.

5 Matteelli A, Rendon A, Tiberi S, et al. Tuberculosis elimination: where are we now? Eur Respir Rev 2018; 27 180035.

6 Zhou G, Luo Q, Luo S, et al. Interferon- $\gamma$ release assays or tuberculin skin test for detection and management of latent tuberculosis infection: a systematic review and meta-analysis. Lancet Infect Dis 2020; 20: 1457-1469.

7 Zellweger JP, Sotgiu G, Corradi M, et al. The diagnosis of latent tuberculosis infection (LTBI): currently available tests, future developments, and perspectives to eliminate tuberculosis (TB). Med Lav 2020; 111: 170-183.

8 Pai M, Denkinger C, Kik S, et al. Gamma interferon release assays for detection of Mycobacterium tuberculosis infection. Clin Microbiol Rev 2014; 27: 3-20.

9 Farhat M, Greenaway C, Pai M, et al. False-positive tuberculin skin tests: What is the absolute effect of BCG and non-tuberculous mycobacteria? Int J Tuberc Lung Dis 2006; 10: 1192-1204.

10 Lalvani A. Diagnosing tuberculosis infection in the 21st century: new tools to tackle an old enemy. Chest 2007; 131: 1898-1906.

11 World Health Organization. WHO guidelines on tuberculosis infection prevention and control, 2019 update. Geneva, World Health Organization, 2019. www.who.int/publications/i/item/9789241550512

12 Barcellini L, Borroni E, Brown J, et al. First evaluation of QuantiFERON-TB gold plus performance in contact screening. Eur Respir J 2016; 48: 1411-1419.

13 Petruccioli E, Vanini V, Chiacchio T, et al. Analytical evaluation of QuantiFERON- Plus and QuantiFERON- Gold In-tube assays in subjects with or without tuberculosis. Tuberculosis (Edinb) 2017; 106: 38-43.

14 Faust L, Ruhwald M, Schumacher S, et al. How are high burden countries implementing policies and tools for latent tuberculosis infection? A survey of current practices and barriers. Health Sci Rep 2020; 3: e158.

15 Fukushima K, Akagi K, Kondo A, et al. First clinical evaluation of the QIAreachTM QuantiFERON-TB for tuberculosis infection and active pulmonary disease. Pulmonology 2021; in press [https://doi.org/10.1016/j. pulmoe.2021.07.003].

16 Stieber F, Howard J, Manissero D, et al. Evaluation of a lateral flow nanoparticle fluorescence assay for TB infection diagnosis. Int J Tuberc Lung Dis 2021; 25: 917-922.

17 Molich R. A critique of "How to specify the participant group size for usability studies: a practitioner's guide" by Macefield. J Usability Studies 2010; 5: 124-128. 